\title{
Including Children with Developmental Disabilities in the Equation During this COVID-19 Pandemic
}

\author{
Ramkumar Aishworiya $^{1}$ [ $\cdot$ Ying Qi Kang ${ }^{1}$
}

Published online: 20 August 2020

(c) Springer Science+Business Media, LLC, part of Springer Nature 2020

\begin{abstract}
Amidst the ongoing novel Coronavirus disease pandemic, children with developmental disabilities warrant specific attention to minimise having disproportionate consequences. These children are especially vulnerable to the effects of the pandemic due to (1) Greater healthcare needs, (2) Dependency on community-based services and (3) Mental health concerns. Healthcare professionals, public health systems and the society needs to come together to advocate for these children by optimising access to healthcare and community intervention services, promoting mental well-being and caregiver welfare. The consequences of missed present-day opportunities might only be evident in the years to come in these children. Hence, despite the prolonged pandemic, with consequent limitations in availability of resources, children with developmental disabilities should continue to be supported.
\end{abstract}

Keywords Developmental delay $\cdot$ COVID-19 $\cdot$ Inequities $\cdot$ Access to care $\cdot$ Children

The ongoing COVID-19 pandemic has highlighted social inequities and disproportionately affected certain groups including nursing home residents, prisoners and migrant workers in the world today. Although COVID-19 amongst children was initially thought to be a mild disease with low incidence, the recent emergence of a possible COVID-19 associated multisystem inflammatory syndrome has reshifted the focus back to children.(Riphagen et al. 2020) However, there is a need to highlight that the pandemic has adverse health outcomes in children beyond the consequence of a viral infection. There has been insufficient attention paid to the mental, social and emotional vulnerability of children during this pandemic. In particular, children with developmental disabilities are especially vulnerable during this unprecedented period of change. In 2016, it was estimated that globally, 52.9 million children younger than 5 years have developmental disabilities.(Olusanya et al. 2018) In the United States of America, 1 in 6 children aged between 3 to 12 years of age have a developmental disability. (Zablotsky et al. 2019). These children and their families,

Ramkumar Aishworiya

aishworiya_ramkumar@nuhs.edu.sg

1 Khoo Teck Puat-National University Children's Medical Institute, National University Health System, Singapore, Singapore have as a group struggled for decades to compete on equal footing across the spectrum of life from education to societal inclusion and peer acceptance with their typically developing peers. As the world struggles to adapt to the ongoing pandemic, special consideration must be accorded to this group of children due to 3 key factors.

\section{Greater Healthcare Needs}

Children with developmental disabilities have higher healthcare needs compared to typically developing children. This includes (1) higher prevalence of other medical conditions such as asthma, food and skin allergies and headaches (2) greater healthcare use and (3) greater burden of their illness on daily-life (such as in mobility and adaptive skills). (Schieve et al. 2012; Oeseburg et al. 2010) Yet these children have greater barriers in accessing healthcare due to an inadequate number of trained specialists, socio-economic status related disparities in access, complex medical comorbidities and barriers of geographical distances.(Soares and Langkamp 2012; Dougherty et al. 2006) Wait times for seeing a developmental behavioral pediatrician (DBP) average between 6 months and 1 year in most countries.(Schieve et al. 2012) These barriers are at risk of being heightened amidst the pandemic due to curtailment of non-critical 
medical care to preserve health system capacities for patients with COVID-19 and to also alleviate the risk of exposure to the virus.

Even as the DBP community endeavors to use telehealth to continue care, this poses its own challenges. Diagnostic evaluation in children with developmental disabilities often require close interaction and rapport building with the child. This is harder to achieve via telemedicine. The time sensitive nature of diagnosis with earlier commencement of early intervention being a consistent outcome predictor, (Hedvall et al. 2015) makes these delays in access to healthcare all the more concerning. Given the lifelong morbidity of developmental disabilities, such delays will arguably be against the best interests of these children.

\section{Dependency on Community-Based Services}

Children with developmental disabilities receive therapy and support from intervention services at specialized centers as well as in schools. Consistency and intensity of intervention is key. This is why children often continue to receive therapy during school vacations. COVID-19 related curtailment of services within the community and school closures limits the provision of continued intervention services, including that of home-based therapy. Video conferencing and telemedicine are being widely used to facilitate learning and therapy from home. However, children with impairments in communication, attention and/or coordination, require specific accommodations to participate and learn effectively. (Ingersoll and Berger 2015) Within the home environment, caregivers may struggle to provide the same level of academic support without the training and expertise of an educator. Children with certain developmental disabilities like autism spectrum disorder (ASD) may also lack the cognitive flexibility to understand that parents are trying to play the role of their teachers or therapists and thus be less compliant. Further, families with limited access to technological infrastructure and the internet, risk being cut off from support services.

\section{Mental Health Concerns}

Children with developmental disabilities may be less resilient to the mental health effects of the pandemic. Mental health disorders such as generalized anxiety disorder, phobias, obsessive compulsive disorder (OCD), mood disorder and psychotic disorder are more common in persons with developmental disabilities compared to the general population.(de Bruin et al. 2007; Merikangas et al. 2015) Evidence has emerged illustrating an increase in levels of anxiety and depression among children in the general population during this pandemic compared to pre-pandemic times.(Duan et al. 2020) Fear of physical illness and home confinement with social isolation were cited as contributing factors. Children with developmental disabilities are likely to be even more vulnerable to the negative mental health consequences of the pandemic as they may have limitations in understanding the rationale for pandemic-related changes such as disruptions to routine and school closures. Specifically, children with OCD are susceptible to an increase in compulsive behaviors such as prolonged hand-washing fueled by the sudden emphasis on personal hygiene and anxiety over disease acquisition; this has recently been shown in adults with OCD.(Shafran et al. 2020; Davide et al. 2020) Further, a lack of access to psychologists and therapists during the pandemic, can contribute to worsening mental health status in these children. This in turn can exacerbate behavioral challenges in these children, adding to the myriad of challenges already faced by caregivers.

Parents of children with developmental disabilities, especially ASD, have greater levels of stress even at baseline, and also a higher divorce rate attributed to the challenges of raising a child with additional needs. (Rivard et al. 2014) These relationships are at risk of further strain when there are mandated alternate work arrangements, financial stressors, uncertainty and lack of childcare support. Restriction of movements between households, with consequent loss of support from extended family members adds to the challenge in parenting. Given that these children are known to have higher rates of maltreatment (Hibbard and Desch 2007), the current confluence of stressors within the family is a further cause for concern.

\section{What Can We Do?}

Addressing the above factors will require coordinated multidisciplinary efforts across national and societal systems, agencies, educational and healthcare institutions. Some of the key mitigating measures will include the following:

1. Maintaining access to outpatient diagnostic and intervention services

It is critical that healthcare facilities that serve children with developmental disabilities (for e.g. DBP clinics, child psychiatry clinics and community based intervention services) be considered essential and remain available. Whenever it is safe to do so, physical visits to these clinics and intervention services should be prioritized. Concurrently, continuing care via telemedicine should be actively explored during the pandemic and even beyond in conjunction with physical visits. Technological infrastructure and training for telemedicine should be facilitated for providers and families. Health- 
care funding to support these aims should be rapidly sanctioned.

2. Facilitating effective learning and therapy

When planning online learning, educators should accommodate children with developmental disabilities with targeted strategies to address their difficulties. For example, children with ASD might require visual schedules, specific communication strategies and additional behavioral support to participate effectively during learning activities. Therapy and/or educational packs containing these materials can be made available online for parents to print or be mailed to families. For children with extremely challenging behaviors, school/centerbased care should be made available as far as possible to provide much needed respite for caregivers and their families.

3. Protecting mental health of children and caregivers

Promoting parental awareness of the mental health needs of their children is important, especially since children may not be able to articulate their distress adequately. Maintaining daily routines including play and recreational activities is crucial. Caregivers can consciously create time and space for recreational physical activities both at home and where possible, outdoors. Help children understand the changes around them, for example by using social stories which can facilitate adjustment to change. Community programs (e.g. parent support groups) can proactively check in on families with children with developmental disabilities. Similarly, physicians can opportunistically discuss children's mental wellbeing, and encourage caregiver self-care and respite.

\section{Conclusion}

Children with developmental disabilities require continued services in a pandemic situation. This ongoing COVID-19 pandemic is unmatched in its sheer global scale of impact with sustained implications for all levels of society. It is akin to a world-wide earthquake with repeated aftershocks lasting for months. The severity and chronicity of COVID-19 will test the resilience and resourcefulness of communities, governments and countries. Children with developmental disabilities are at risk of being lost amidst the hubbub of the pandemic. Parents of children with disabilities have traditionally been the sole advocates for their children. During this pandemic where parents themselves are struggling more than ever, education, health, early intervention and social services must keep these children in focus and collaborate to meet the challenges unique to this population of children. The missed opportunities of serving children with developmental disabilities would only manifest in years to come.
During this unprecedented time for humanity, pediatricians must come together to care and advocate for nations and communities to include children with developmental disabilities in the equation.

Acknowledgments We would like to thank Dr Dimple Rajgor for helping with reviewing, editing, formatting, and submission of the manuscript for publication.

Author Contributions Dr. RA and Dr. YQK both conceptualized and wrote the initial manuscript, and subsequently reviewed and revised it.

Funding None.

\section{Compliance with Ethical Standards}

Conflict of interest The authors declare that they have no conflict of interest.

Ethical Approval This article does not contain any studies with human participants or animals performed by any of the authors.

\section{References}

Davide, P., Andrea, P., Martina, O., Andrea, E., Davide, D., \& Mario, A. (2020). The impact of the COVID-19 pandemic on patients with OCD: Effects of contamination symptoms and remission state before the quarantine in a preliminary naturalistic study. Psychiatry Research. https://doi.org/10.1016/j.psychres.2020.113213.

de Bruin, E. I., Ferdinand, R. F., Meester, S., de Nijs, P. F. A., \& Verheij, F. (2007). High rates of psychiatric co-morbidity in PDDNOS. Journal of Autism and Developmental Disorders, 37(5), 877-886. https://doi.org/10.1007/s10803-006-0215-x.

Dougherty, D., Simpson, L. A., \& McCormick, M. C. (2006). Rural areas and children's health care coverage, use, expenditures, and quality: Policy implications. Ambulatory Pediatrics, 6(5), 265-267.

Duan, L., Shao, X., Wang, Y., Huang, Y., Miao, J., Yang, X., et al. (2020). An investigation of mental health status of children and adolescents in China during the outbreak of COVID-19. Journal of Affective Disorders, 275, 112-118.

Hedvall, A., Westerlund, J., Fernell, E., Norrelgen, F., Kjellmer, L., Olsson, M. B., et al. (2015). Preschoolers with autism spectrum disorder followed for 2 years: Those who gained and those who lost the most in terms of adaptive functioning outcome. Journal of Autism and Developmental Disorders, 45(11), 3624-3633. https ://doi.org/10.1007/s10803-015-2509-3.

Hibbard, R. A., \& Desch, L. W. (2007). Maltreatment of children with disabilities. Pediatrics, 119(5), 1018-1025.

Ingersoll, B., \& Berger, N. I. (2015). Parent engagement with a telehealth-based parent-mediated intervention program for children with autism spectrum disorders: Predictors of program use and parent outcomes. Journal of Medical Internet Research, 17(10), e227.

Merikangas, K. R., Calkins, M. E., Burstein, M., He, J.-P., Chiavacci, R., Lateef, T., et al. (2015). Comorbidity of physical and mental disorders in the neurodevelopmental genomics cohort study. Pediatrics, 135(4), e927-e938.

Oeseburg, B., Jansen, D., Dijkstra, G., Groothoff, J., \& Reijneveld, S. (2010). Prevalence of chronic diseases in adolescents with 
intellectual disability. Research in Developmental Disabilities, 31(3), 698-704.

Olusanya, B. O., Davis, A. C., Wertlieb, D., Boo, N.-Y., Nair, M., Halpern, R., et al. (2018). Developmental disabilities among children younger than 5 years in 195 countries and territories, 1990-2016: A systematic analysis for the Global Burden of Disease Study 2016. The Lancet Global Health, 6(10), e1100-e1121.

Riphagen, S., Gomez, X., Gonzalez-Martinez, C., Wilkinson, N., \& Theocharis, P. (2020). Hyperinflammatory shock in children during COVID-19 pandemic. The Lancet, 395(10237), 1607-1608.

Rivard, M., Terroux, A., Parent-Boursier, C., \& Mercier, C. (2014). Determinants of stress in parents of children with autism spectrum disorders. Journal of Autism and Developmental Disorders, 44(7), 1609-1620.

Schieve, L. A., Gonzalez, V., Boulet, S. L., Visser, S. N., Rice, C. E., Braun, K. V. N., et al. (2012). Concurrent medical conditions and health care use and needs among children with learning and behavioral developmental disabilities, National Health Interview Survey, 2006-2010. Research in Developmental Disabilities, 33(2), 467-476.
Shafran, R., Coughtrey, A., \& Whittal, M. (2020). Recognising and addressing the impact of COVID-19 on obsessive-compulsive disorder. The Lancet Psychiatry, 7(7), 570-572.

Soares, N. S., \& Langkamp, D. L. (2012). Telehealth in developmentalbehavioral pediatrics. Journal of Developmental \& Behavioral Pediatrics, 33(8), 656-665.

Zablotsky, B., Black, L. I., Maenner, M. J., Schieve, L. A., Danielson, M. L., Bitsko, R. H., et al. (2019). Prevalence and trends of developmental disabilities among children in the United States: 20092017. Pediatrics, 144(4), e20190811. https://doi.org/10.1542/ peds.2019-0811.

Publisher's Note Springer Nature remains neutral with regard to jurisdictional claims in published maps and institutional affiliations. 